\title{
MRI is a state of art imaging modality in characterization of indeterminate adnexal masses
}

\author{
Amit Nandan Dhar Dwivedi ${ }^{1 *}$, Shivi Jain ${ }^{1}$, Ram Chandra Shukla ${ }^{1}$, Madhu Jain ${ }^{2}$, Arvind Srivastava ${ }^{1}$, \\ Ashish Verma ${ }^{1}$ \\ ${ }^{1}$ Department of Radiodiagnosis and Imaging, Institute of Medical Science, Varanasi, India \\ ${ }^{2}$ Department of Obstetrics and Gynecology, Institute of Medical Science, Varanasi, India \\ Email: ${ }^{*}$ amitnandan21@yahoo.com
}

Received 19 December 2012; revised 23 January 2013; accepted 29 January 2013

\section{ABSTRACT}

Aims and Objectives: The aim of this diagnostic observational study was to find an association of final diagnosis of adnexal masses suggested by MRI and compared it as an imaging modality in determining the origin, nature (benign/malignant) \& characteristics of adnexal masses by calculating sensitivity, specificity, and diagnostic accuracy. Materials and Methods: The present study was carried out in 90 patients in the department of radio diagnosis and imaging, institute of medical sciences, Banaras Hindu University (BHU). The patients were referred from department of obstetrics \& gynecology, institute of medical sciences. Majority of the referred cases were those who had clinical features of abdominal pain, abdominal lump, menstrual irregularity, ascites, and anorexia or weight loss $\&$ in whom adnexal mass was suspected clinically. Magnetic resonance imaging was performed using 1.5 Tesla MR Scanner, Magnetom Avanto (Siemens Healthcare). Results: Out of 114 masses, $17(14.9 \%)$ were malignant. The benign adnexal masses were maximum in the age group 20 39 years $(56 / 97,57.7 \%)$, while malignant masses were mainly found in women $\geq \mathbf{6 0}$ years of age (11/17, $64.7 \%)$. CA-125 level was grossly elevated in association with $35.3 \%$ of the malignant masses. On MRI, the sensitivity for the mass of ovarian origin was $(97.7 \%)$ and specificity was $(73.1 \%)$. The diagnostic accuracy was $(92.1 \%)$. The mass of uterine origin had a sensitivity of $(\mathbf{7 3 . 1 \% )}$ and diagnostic accuracy (99.1\%). Conclusion: MRI, because of its accuracy in identifying the origin of adnexal mass and characterizing the solid, hemorrhagic, fatty and fibrous content, may obviate surgery or significantly contribute to preoperative planning for a sonographically indeterminate mass. MRI is the state of the art imaging modality for evaluation of adnexal masses with an over-

"Corresponding author. all high diagnostic accuracy.

Keywords: MRI; Adnexal Masses; Diagnostic Accuracy

\section{INTRODUCTION}

Adnexal masses are quite common. An estimated 5\% to $10 \%$ of women undergo surgery for suspicious adnexal masses, less than $25 \%$ of which prove to be malignant (NIH Consensus Conference. Ovarian cancer. Screening, treatment, and follow-up. NIH Consensus Development Panel on Ovarian Cancer). Exclusion of malignancy in an adnexal mass is of paramount importance. It is the most crucial step after identification of a mass and has a profound effect on the patient's management. It is all the more important to know the nature of the tumor before surgery in a young woman. The benign or malignant nature of a clinically diagnosed adnexal mass is frequently not evident before surgical exploration and histological examination. A reliable method to differentiate benign from malignant adnexal masses would allow for appropriate subspecialty referral, optimal preoperative planning and counselling of the patient. Ultrasonography (US) is the accepted primary imaging technique for evaluating adnexal masses. However, the overlap in USG features of benign or malignant pelvic masses warrant further research on complimentary techniques. Colour Doppler imaging has been investigated as possible means of improving the specificity of gray scale sonography in differentiating benign from malignant lesions. The sensitivity of morphological analysis with US in predicting malignancy has been reported to be $85 \%$ to $97 \%$ whereas the specificity ranges from $56 \%$ to $95 \%[1,2]$. For Doppler US techniques, sensitivities of $50 \%$ to $100 \%$ and specificities of $46 \%$ to $100 \%$ have been reported. Despite the claim of improvement in sensitivity and specificity with combined approach, some adnexal masses remain indeterminate. Magnetic resonance imaging (MRI) has been shown to have potential for characterizing adnexal 
masses $[3,4]$. These studies have shown that MRI is superior in identifying endometriomas, dermoids and fibroids, and the reproducibility and accuracy have been documented. Recent literature search reveals multiple MRI features specific for ovarian malignancy. Therefore, in the present study, we have tried to assess the ability of MRI to characterize indeterminate adnexal masses. MRI might offer a non-invasive and more accurate characterization of the doubtful masses.

\section{MATERIALS AND METHODS}

The present study was carried out in 90 patients in the Department of Radio diagnosis and Imaging and Department of Obstetrics \& Gynecology, Institute of Medical Sciences Banaras Hindu University Varanasi, India, in accordance with the declaration of Helsinki guidelines on good clinical practice. The Institutional ethical committee approved the study and written informed consent was obtained from each patient. Majority of the referred cases were those who had clinical features of abdominal pain, abdominal lump, menstrual irregularity, ascites, and anorexia or weight loss \& in whom adnexal mass was suspected clinically. Magnetic resonance imaging was performed using 1.5 Tesla MR Scanner, Magnetom Avanto (Siemens Healthcare). Clinical assessment included age, pregnancy status, menopausal status and CA125 levels (if done) of each patient. Clinical history was elicited from the patients or guardians. Clinical records were evaluated to look for any relevant investigation and for any previous operative procedure, or histopathological examination. All the patients undergoing MRI had fast for 4 hours and received an anti-spasmodic intramuscularly 10 minutes before MRI to reduce bowel peristalsis and to improve visualization of adnexa and peritoneal surfaces. Image acquisition was done in axial, sagittal \& coronal planes in T1 \& T2-weighted sequences using 8 channels transmit-receive torso phased-array coil. Fat-suppressed T1 weighted imaging was done when mass showed high signal intensity on $\mathrm{T} 1 \mathrm{~W}$ sequence to confirm/rule out presence of fat. The fields of view were small $(20 \mathrm{~cm})$ with high resolution matrixes $(256 \times 256)$, and thin slices of $4 \mathrm{~mm}$.

\subsection{Image Analysis}

MRI assessed the criteria of size of mass, multiplicity \& laterality, thickness of wall \& septa, papillary excrescences/vegetations, tissue content, metastasis, ascites, lymphadenopathy, involvement of pelvic organs \& sidewalls based on same parameters. MRI criteria were taken according to Stevens et al. \& Ferrazzi et al. $(2005)[5,6]$. Only non-contrast MRI was done. Contrast-MRI was not done as Hricak et al. [3] did not find any significant difference in the rate of detection of benign \& malignant lesions between non-enhanced \& contrast-enhanced images. MRI also assessed absence/presence of necrosis in solid lesion, and signal intensities on T1-weighted (fatsuppressed \& non fat-suppressed) \& T2-weighted sequences. All patients with cardiac-pacemaker implants, aneurysmal clips, metallic implants and ferrous foreign bodies were excluded from the study group. The final diagnosis was established by clinical follow-up, imaging follow-up, per-operative findings and/or histopathology.

\subsection{Statistical Analysis}

Statistical analysis was applied to find out the sensitivity, specificity, positive predictive value, negative predictive value and diagnostic accuracy. Chi-square $\left(\chi^{2}\right)$ test was used to find out the significant association between the variables. The level of significance was taken as $5 \%$ at two-tailed test. Agreement between MRI and final diagnosis was established using kappa statistics \& 95\% CIs. The data analysis was done using SPSS (version 16) system.

\section{RESULTS}

There were 90 patients with 114 adnexal masses. They were single in 66, bilateral in 16 and unilateral dual masses in 8 patients. Their age ranged from 12 years to 65 years. Out of 114 masses, 17 (14.9\%) were malignant. The benign adnexal masses were maximum in the age group 20 - 39 years $(56 / 97,57.7 \%)$, while malignant masses were mainly found in women $\geq 60$ years of age $(11 / 17,64.7 \%)$. (Table 1) CA-125 level was grossly elevated in association with $35.3 \%$ of the malignant masses. On MRI, the sensitivity for the mass of ovarian origin was $(97.7 \%)$ and specificity was (73.1\%), (Figures 1 and 2). The diagnostic accuracy was $(92.1 \%)$. The mass of uterine origin had a sensitivity of $(73.1 \%)$ and diagnostic accuracy (99.1\%) on MRI, (Figure 3). There were seven patients in "others" category, four of them had encysted ascites and three had paraovarian cysts much larger than coexisting hemorrhagic ovarian cysts. The encysted ascites was misinterpreted as benign ovarian cyst. The

Table 1. Age distribution of adnexal masses.

\begin{tabular}{ccccc}
\hline & \multicolumn{4}{c}{ Final Diagnosis } \\
\cline { 2 - 5 } Age (Years) & \multicolumn{2}{c}{ Benign } & \multicolumn{2}{c}{ Malignant } \\
\cline { 2 - 5 } & No. & $\%$ & No. & $\%$ \\
\hline$<20$ & 33 & 34.0 & 0 & 0 \\
$20-39$ & 56 & 57.7 & 2 & 11.8 \\
$40-59$ & 7 & 7.2 & 4 & 23.5 \\
$\geq 60$ & 1 & 1.0 & 11 & 64.7 \\
Total & 97 & 100 & 17 & 100 \\
\hline
\end{tabular}




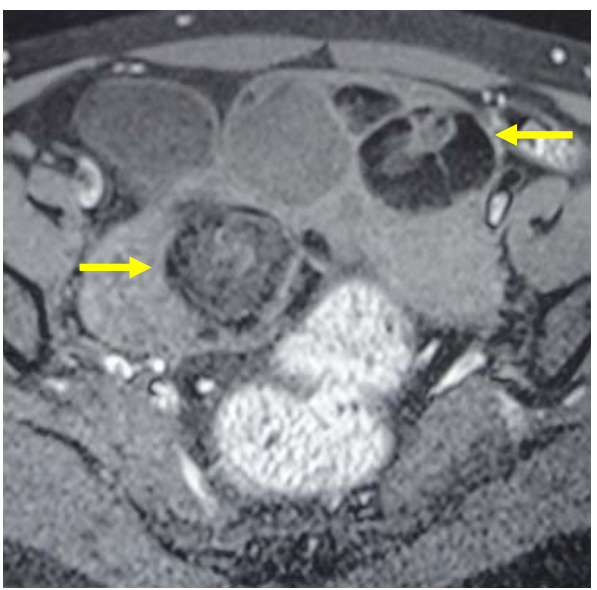

Figure 1. Bilateral mature cystic teratomas: Axial T1-weighted fat-suppressed image shows bilateral adnexal masses (yellow arrows) with suppressed high signal indicating presence of fat.



Figure 2. Endometrioma: Axial T2-weighted image shows dependent layering with relatively hypointense fluid-fluid level (yellow arrow).

paraovarian cyst coexisting with hemorrhagic ovarian cyst was interpreted as two different lesions but within the same ovary. MRI had maximum accuracy (96.5\%) and agreement ( $\kappa: 0.85, \mathrm{CI}: 0.67-0.85)$ in the diagnosis of a malignant ovarian tumor on the basis of criteria such as tumor size $>4 \mathrm{~cm}$, necrosis within the tumor, wall thickness, septal thickness and tissue content (Figure 4). Therefore all the patients in young age group especially with normal CA-125 should be evaluated by MRI to exclude malignancy and to save the patients form unnecessary laparotomy or radical operation. Endometriomas were most accurately diagnosed by MRI with $100 \%$ sensitivity and accuracy. MRI was the best imaging modality to identify fibroids with $83.3 \%$ sensitivity, $100 \%$ specificity and $99.1 \%$ accuracy. Thus, the pedicle of fibroid, and fibrous tissue nature of fibromyomas can be best delineated by MRI. The advantage of MRI lies in its

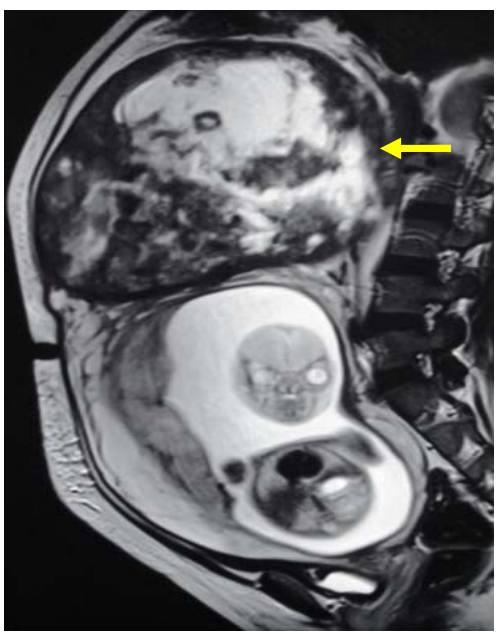

Figure 3. Uterine fibroid with pregnancy mimicking malignant mass on imaging: Sagittal T2-weighted image shows supraumbilical large solid-cystic mass with apparent minimal extension along mesenteric vessels raising suspicion of mesenchymal malignant tumour (yellow arrow).

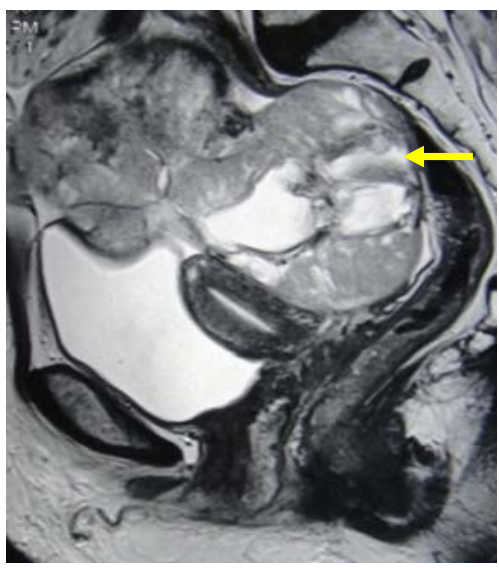

Figure 4. Malignant ovarian mass: Sagittal T2-weighted image shows cystic mass with solid areas raising suspicion of malignancy (yellow arrow).

ability to reveal normal ovaries and to delineate the pathognomic tortuous folds of a hydrosalpinx.

\section{DISCUSSION}

In a screening of postmenopausal, high-risk population, the prevalence of ovarian cancer was approximately $0.1 \%$, and the prevalence of pathology proven benign masses ranged from $0.8 \%$ to $1.8 \%$ [7-10]. Our study outlines the typical magnetic resonance imaging features of the most commonly encountered adnexal lesions. The American College of Radiology has provided guidelines to assist in the appropriate utilization of MRI in the pel- 
vis (ACR Practice Guideline for the Performance of Magnetic Resonance Imaging (MRI) of the Soft Tissue Components of the Pelvis). MRI is recommended for the characterization of complex adnexal masses that are indeterminate on US. Most studies have shown MRI to be superior to endovaginal US in the differentiation of benign from malignant adnexal lesions [11-14]. Studies have reported sensitivity from $67 \%$ to $100 \%$ and specificity to range between $77 \%$ and $100 \%$ [15-20]. Preliminary studies have demonstrated the value of dynamic contrast-enhanced MRI in the characterization of ovarian epithelial tumors $[21,22]$. The use of diffusion-weighted imaging is debatable with no significant difference between malignant and benign adnexal lesions [23]. The additional benefit of MRI for characterization of ovarian masses in selected patients has been well documented [24-27]. A prospective study of 103 women with adnexal masses found that MRI had sensitivity and specificity of $96 \%$ and $100 \%$ for diagnosis of pedunculated leiomyomas, $100 \%$ and $99 \%$ for dermoid cyst, and $92 \%$ and $91 \%$ for endometriomas [25]. In a study of women with a variety of gynecologic diseases, including adnexal masses, pelvic MRI was shown to alter treatment in up to $73 \%$ of patients, decrease the number of invasive surgeries, and reduce overall expenditures for care [28]. In our study, MRI significantly improved the specificity in deciding the ovarian origin of adnexal mass with higher predictive values and diagnostic accuracy. Magnetic resonance imaging is a valuable modality for non-invasive evaluation of the adnexal masses for elucidating the nature and origin of variety of pathological conditions because of excellent spatial and tissue contrast resolution, good tissue characterization and multiplanar imaging capability without any risk of ionizing radiation [29]. MRI has shown to have a $91 \%$ - 93\% overall accuracy for differentiating benign from malignant tumors [4]. Ahmad et al., [29] consider MRI to be the state of the art imaging modality for adnexal masses with high overall diagnostic accuracy besides aiding in staging of malignant adnexal masses. Out of 114 masses that we evaluated, 17 were malignant. Pre-operative determination of malignant nature of the mass assumes great significance among young women with normal CA-125 level as the further line of management would alter their obstetrical prospects as well. The benign nature of the mass in our patients was determined by MRI with $53 \%$ sensitivity, $98 \%$ specificity and $91 \%$ accuracy while it was determined to be malignant with $76.5 \%$ sensitivity, $100 \%$ specificity and $96.5 \%$ accuracy. It also had excellent agreement $(\kappa=0.85)$. According to Adusumilli et al., [30] the specificity of MRI for correctly making a benign diagnosis was $94 \%$ (98\% by us) and its sensitivity for correctly identifying a malignant lesion was $100 \%(95 \%$ CI, $57 \%-100 \%)$ compared with $76.5 \%$ found by us. There were four masses in our study, two of ovarian pregnancy and two of ovarian torsion with hemorrhage. The ovarian pregnancy was misinterpreted as tubal pregnancy because ovarian follicles were found at the periphery of gestation sac giving an impression of normal ovary. However, MRI showed an eccentric solid mural nodule (size $>3.0 \mathrm{~mm}$ ) and labeled it as indeterminate as it could not authentically comment upon the nature of the mass. The eccentric nodule turned out to be a twisted pedicle per-operatively. Our findings are quite consistent with most of the other studies. Our findings also suggest like those of others that MRI should have selective application for sonographically labeled complex masses and not be used at random.

\section{CONCLUSION}

MRI, because of its accuracy in identifying the origin of adnexal mass and characterizing the solid, hemorrhagic, fatty and fibrous content, may obviate surgery or significantly contribute to preoperative planning for a sonographically indeterminate mass. MRI is the state of the art imaging modality for evaluation of adnexal masses with an overall high diagnostic accuracy.

\section{REFERENCES}

[1] Ferrazzi, E., Zanetta, G., Dordoni, D., et al. (1997) Transvaginal ultrasonographic characterization of ovarian masses: Comparison of five scoring systems in a multicenter study. Ultrasound in Obstetrics and Gynecology, 10, 192-197. doi:10.1046/j.1469-0705.1997.10030192.x

[2] Kurjak, A. and Predanic, M. (1992) New scoring system for prediction of ovarian malignancy based on transvaginal color Doppler sonography. Journal of Ultrasound in Medicine, 11, 631-638.

[3] Hricak, H., Chen, M., Coakley, F.V., KinKel, K., Sica, G., et al. (2000) Complex adnexal masses: Detection and characterization with MRI-Multivariate analysis. Radiology, 214, 39-46.

[4] Sohaib, S.A., Sahdev, A., Van Trappen, P.O., Jacobs, I.J. and Reznek, R.H. (2003) Characterization of adnexal mass lesions on MRI. American Journalism Review, 180, 1297-1304.

[5] Stevens, S.K., Hricak, H. and Stern, J.L. (1991) Ovarian lesions: Detection and characterization with gadoliniumenhanced MRI at 1.5 T. Radiology, 181, 481-488.

[6] Ferrazzi, E., Lissoni, A.A., Dordoni, D., Trio, D., Redaelli, L., et al. (2005) Differentiation of small adnexal masses based on morphologic characteristics of transvaginal sonographic imaging: A multicentric study. Journal of Ultrasound in Medicine, 24, 1467-1473.

[7] Dorum, A., Blom, G.P., Ekerhovd, E., et al. (2005) Prevalence and histologic diagnosis of adnexal cysts in postmenopausal women: An autopsy study. American Journal of Obstetrics and Gynecology, 192, 48-54. 


\section{doi:10.1016/j.ajog.2004.07.038}

[8] Hilger, W.S., Magrina, J.F. and Magtibay, P.M. (2006) Laparoscopic management of the adnexal mass. Clinical Obstetrics and Gynecology, 49, 535-548. doi:10.1097/00003081-200609000-00013

[9] Modesitt, S.C., Pavlik, E.J., Ueland, F.R., et al. (2003) Risk of malignancy in unilocular ovarian cystic tumors less than 10 centimeters in diameter. Obstetrics and Gynecology, 102, 594-599. doi:10.1016/S0029-7844(03)00670-7

[10] Myers, E.R., Bastian, L.A., Havrilesky, L.J., et al. (2006) Management of adnexal mass. Evidence Report/Technology Assessment, 130, 1-145.

[11] Yamashita, Y., Toashima, M., Hatanaka, Y., et al. (1995) Adnexal masses: Accuracy of characterization with transvaginal US and precontrast and postcontrast MRI. Radiology, 194, 557-565.

[12] Komatsu, T., Konishi, I., Mandai, M., Togashi, K., Kawakami, S., Konishi, J., et al. (1996) Adnexal masses: Transvaginal US and gadolinium-enhanced MRI assessment of intratumoral structure. Radiology, 198, 109-115.

[13] Hata, K., Hata, T., Manabe, A., et al. (1992) A critical evaluation of transvaginal Doppler studies, transvaginal sonography, magnetic resonance imaging, and CA 125 in detecting ovarian cancer. Obstetrics and Gynecology, 80, 922-926.

[14] Yamashita, Y., Hatanaka, Y., Torashima, M., Takahashi, M., Miyazaki, K. and Okamura, H. (1997) Characterization of sonographically indeterminate ovarian tumors with MR imaging: A logistic regression analysis. Acta Radiologica, 38, 572-577.

[15] Buist, M.R., Golding, R.P., Burger, C.W., et al. (1994) Comparative evaluation of diagnostic methods in ovarian carcinoma with emphasis on CT and MRI. Obstetrics and Gynecology, 52, 191-198.

[16] Grab, D., Flock, F., Stohr, I., et al. (2000) Classification of asymptomatic adnexal masses by ultrasound, magnetic resonance imaging, and positron emission tomography. Obstetrics and Gynecology, 77, 454-459.

[17] Huber, S., Medl, M., Baumann, L., et al. (2002) Value of ultrasound and magnetic resonance imaging in the preoperative evaluation of suspected ovarian masses. Anticancer Research, 22, 2501-2507.

[18] Jain, K.A., Friedman, D.L., Pettinger, T.W., et al. (1993) Adnexal masses: Comparison of specificity of endovaginal US and pelvic MR imaging. Radiology, 186, 697-704.

[19] Sohaib, S.A., Mills, T.D., Sahdev, A., et al. (2005) The role of magnetic resonance imaging and ultrasound in patients with adnexal masses. Clinical Radiology, 60, 340348. doi:10.1016/j.crad.2004.09.007
[20] Kurtz, A.B., Tsimikas, J.V., Tempany, C.M.C., Hamper, U.M., Arger, P.H., Bree, R.L., et al. (1999) Diagnosis and staging of ovarian cancer: Comparative values of Doppler and conventional US, CT, and MRI correlated with surgery and histopathologic analysis-Report of the radiology diagnostic oncology group. Radiology, 212, 19-27.

[21] Thomassin-Naggara, I., Bazot, M., Darai, E., et al. (2008) Epithelial ovarian tumors: Value of dynamic contrastenhanced MR imaging and correlation with tumor angiogenesis. Radiology, 248, 148-159. doi:10.1148/radiol.2481071120

[22] Thomassin-Naggara, I., Darai, E., Cuenod, C.A., et al. (2008) Dynamic contrast enhanced magnetic resonance imaging: A useful tool for characterizing ovarian epithelial tumors. Journal of Magnetic Resonance Imaging, 28, 111-120. doi:10.1002/jmri.21377

[23] Fujii, S., Kakite, S., Nishihara, K., et al. (2008) Diagnostic accuracy of diffusion weighted imaging in differentiating benign from malignant ovarian lesions. Journal of Magnetic Resonance Imaging, 28, 1149-1156. doi:10.1002/jmri.21575

[24] Outwater, E.K. and Dunton, C.J. (1995) Imaging of the ovary and adnexa: Clinical issues and applications of MRI. Radiology, 194, 1-18.

[25] Scoutt, L.M., McCarthy, S.M., Lange, R., Bourque, A. and Schwartz, P.E. (1994) MR evaluation of clinically suspected adnexal masses. Journal of Computer Assisted Tomography, 18, 609-618. doi:10.1097/00004728-199407000-00019

[26] Yamashita, Y., Torashima, M., Hatanaka, Y., Harada, M., Higashida, Y., Takahashi, M., et al. (1999) Adnexal masses: Accuracy of characterization with transvaginal US and precontrast and postcontrast MRI. Radiology, 194, 557565.

[27] Stevens, S.K., Hricak, H. and Campos, Z. (1993) Teratomas versus cystic hemorrhagic adnexal lesions: Differentiation with proton-selective fat-saturation MRI. Radiology, 186, 481-488.

[28] Schwartz, L.B., Panageas, E., Lange, R., Rizzo, J., Comite, F. and McCarthy, S. (1994) Female pelvis: Impact of MRI on treatment decisions and net cost analysis. Radiology, 192, 55-60.

[29] Ahmad, I., Kirmani, S., Rashid, M. and Ahmad, K. (2011) MR imaging of adnexal masses: A review. NJR, 1, 5460.

[30] Adusumilli, S., Hussain, H.K., Caoili, E.M., Weadock, W.J., Murray, J.P., Johnson, T.D., Chen, Q. and Desjardins, B. (2006) MRI of sonographically indeterminate adnexal masses. American Journal of Roentgenology, 187, 732-740. doi:10.2214/AJR.05.0905 